Vol. 42 (1990) [145-152]

\title{
RINGS WHOSE ADDITIVE ENDOMORPHISMS ARE RING ENDOMORPHISMS
}

\author{
Gary Birkenmeier and Henry Heatherly
}

\begin{abstract}
A ring $R$ is said to be an $A E$-ring if every additive endomorphism is a ring endomorphism. In this paper further steps are made toward solving Sullivan's Problem of characterising these rings. The classification of $A E$-rings with $R^{3} \neq 0$ is completed. Complete characterisations are given for $A E$-rings which are either: (i) subdirectly irreducible, (ii) algebras over fields, or (iii) additively indecomposable. Substantial progress is made in classifying $A E$-rings which are mixed - the last open case - by imposing various finiteness conditions (chain conditions on special ideals, height restricting conditions). Several open questions are posed.
\end{abstract}

\section{INTRODUCTION}

A ring is said to be an $A E$-ring if every additive endomorphism is a ring endomorphism [4]. Sullivan [10] posed the problem of classifying $A E$-rings. Kim and Roush [8] classified finite $A E$-rings and Feigelstock [4] classified torsion $A E$-rings and gave several useful properties of $A E$-rings in general. Dhompongsa and Sanwong considered nonnil $A E$-rings [3]. In this paper we finish the characterisation of $A E$-rings with $R^{3} \neq 0$ and turn to $A E$-rings with $R^{3}=0, R^{2} \neq 0$, and which are not torsion, but which do have an element of order two. This is the last remaining open part of the Sullivan Problem. Necessary conditions are given for such rings under various finiteness hypotheses (chain conditions and height conditions). Complete classifications are given for $A E$-rings which are (i) subdirectly irreducible (ii) algebras over a field or (iii) additively indecomposable. An example is given which answers in the negative a question raised by Feigelstock [4]. It is shown that the class of $A E$-rings is properly contained in the class of self distributive rings $[2,9]$. It is of interest to note that the concept of an $A E$-ring has been generalised along two completely different lines $[5,1]$. We conclude the paper with four open questions.

The notation and terminology used herein will be that found in Fuchs [6], unless otherwise noted. We use $C(m)$ to denote the cyclic group of order $m$ and $C\left(p^{\infty}\right)$ for

Received 28 September 1989

Part of this work was completed while the first author had a faculty research grant from the University of Southwestern Louisiana. The authors wish to thank Professors S. Feigelstock and R. Raphael for their comments.

Copyright Clearance Centre, Inc. Serial-fee code: 0004-9729/90 \$A2.00+0.00. 
the quasicyclic group for prime $p$. The ring of integers is denoted by $Z$ and the ring of integers modulo $m$ by $Z /(m)$. For any ring $R$, we use $R^{+}$for the additive group of $R, R_{p}$ for the set (ideal) of all elements whose orders are a power of the prime $p$, and $T\left(R^{+}\right)$for the torsion subgroup of $R^{+}$. The ring of rational real numbers is denoted by $Q$. For any set $S,|S|$ is the cardinality of $S$.

Useful in the sequel are the following results for an $A E$-ring $R$, each found in Feigelstock [4].

(1) If $R^{+}=H^{+} \oplus K^{+}$, then $R=H \oplus K$ and each of the ideals $H$ and $K$ are $A E$-rings.

(2) $2 R^{2}=0$ and if $R^{2} \neq 0$, then $R_{2}^{+}$is reduced.

From (1) we immediately have that the maximal divisible subgroup $D$ of $R^{+}$is an $A E$-ring and $R=D \oplus A$, where $A^{+}$is reduced. From (2) we see that the elements of odd order are in Ann $R$, the two-sided annihilator of $R$; and if $R_{2}=0$, then $R^{2}=0$.

\section{MAIN RESUlts}

Lemma 1. Let $R$ be an $A E$-ring and $f, g \in$ End $\left(R^{+}\right)$.

(i) If $x, y \in R$, then $f(x) g(y)=g(x) f(y)$.

(ii) $(\operatorname{Im} f)(\operatorname{ker} f)=0=(\operatorname{ker} f)(\operatorname{Im} f)$.

Proof: (i)

$$
\begin{aligned}
f(x) f(y) & +g(x) g(y)=f(x y)+g(x y)=[f+g](x y) \\
& =([f+g](x))([f+g](y))=(f(x)+g(x))(f(y)+g(y)) \\
& =f(x) f(y)+f(x) g(y)+g(x) f(y)+g(x) g(y) .
\end{aligned}
$$

So

$$
0=f(x) g(y)+g(x) f(y),
$$

and

$$
f(x) g(y)=g(x) f(y)
$$

since

$$
2 R^{2}=0 \text {. }
$$

(ii) Let $x \in \operatorname{ker} f$ and $y \in R$. Then $f(y) x=f(y) i(x)=i(y) f(x)=0$ where $i$ is the identity endomorphism. Similarly, $(\operatorname{ker} f)(\operatorname{Im} f)=0$.

Lemma 2. Let $R$ be an $A E$-ring; $R=B \oplus X$, and $g: X \rightarrow B$ is an additive homomorphism. Then

(i) $g(X) \cdot R=0=R \cdot g(X)$;

(ii) if $g$ is onto, then $B^{2}=R B=B R=0$;

(iii) if $g$ is one-to-one, then $X^{2}=X R=R X=0$. 
Proof: Define $f, \pi \in \operatorname{End}\left(R^{+}\right)$by $f(b+x)=g(x), \pi(b+x)=b$, where $b \in B$ and $x \in X$.

(i) Let $g(x) \in g(X)$. From Lemma $1, g(x) b=f(x) \pi(b)=\pi(x) f(b)=0$. Thus $g(X) \cdot B=0=g(X) \cdot R$. Similarly, $R \cdot g(X)=0$.

(ii) This part follows from (i).

(iii) Let $x, y \in X$. Then $g(x y)=f(x y)=f(x) f(y)=g(x) f(y) \in g(X) \cdot R=$ 0 . Thus $x y=0$.

Crucial to the theory developed herein (and in [4]) is the following class of rings.

EXAMPLE 3. On the additive group of order $2^{n}, n>0$, with generator $y$, define multiplication via $\left(m_{1} y\right)\left(m_{2} y\right)=2^{n-1} m_{1} m_{2} y$. We call this ring the fundamental $A E$. ring of type $n$ and denote it by $F S(n)$. Note $F S(1) \cong Z /(2)$ and for $n>1,(F S(n))^{3}=$ 0 .

Theorem 4. Let $R$ be a ring with $R^{2} \neq 0$ and $R_{2}$ a direct summand of $R$. Then $R$ is an $A E$-ring if and only if:

(i) $R_{2}=C \oplus S$, where $C \cong F S(n)$ and $2^{n-1} S=0=S^{2}, n \geqslant 1$.

(ii) $R=R_{2} \oplus N$, as a ring direct sum, with $N^{2}=0$, and

(iii) if $g \in \operatorname{Hom}\left(N^{+}, R_{2}^{+}\right)$, then $(g(N)) \cdot R_{2}=0$.

Proof: Assume (i)-(iii) hold. Note $R$ is commutative. Let $f \in \operatorname{End}\left(R^{+}\right)$. Since $R_{2}$ is fully invariant in $R^{+}$and $R_{2}$ is an $A E$-ring [4, Theorem 6], we have that $f$ restricted to $R_{2}$ is a ring endomorphism. For convenience, let $B=R_{2}$. For each $n, n^{\prime} \in N, b \in B$, we have:

$$
b \cdot f(n)=b \cdot\left(\pi_{B} f(n)+\pi_{N} f(n)\right)=0
$$

and

$$
\begin{aligned}
f(n) \cdot f\left(n^{\prime}\right) & =f(n) \cdot\left(\pi_{B} f\left(n^{\prime}\right)+\pi_{N} f\left(n^{\prime}\right)\right)=f(n) \cdot \pi_{N} f\left(n^{\prime}\right) \\
& =\left(\pi_{B} f(n)+\pi_{N} f(n)\right) \cdot \pi_{N} f\left(n^{\prime}\right)=0
\end{aligned}
$$

where $\pi_{B}$ and $\pi_{N}$ are the projection mappings onto $B$ and $N$, respectively. For each $n_{1}, n_{2} \in N, b_{1}, b_{2} \in B$, we have:

$$
f\left(\left(b_{1}+n_{1}\right)\left(b_{2}+n_{2}\right)\right)=f\left(b_{1} b_{2}+n_{1} n_{2}\right)=f\left(b_{1} b_{2}\right)+f(0)=f\left(b_{1}\right) f\left(b_{2}\right),
$$

and

$$
\left(f\left(b_{1}+n_{1}\right)\right) \cdot\left(f\left(b_{2}+n_{2}\right)\right)=\left(f\left(b_{1}\right)+f\left(n_{1}\right)\right) \cdot\left(f\left(b_{2}\right)+f\left(n_{2}\right)\right)=f\left(b_{1}\right) f\left(b_{2}\right) .
$$


Conversely, assume $R$ is an $A E$-ring. From [4, Lemma 3 and Theorem 6], we have (i) and (ii), while (iii) follows from Lemma 2 above.

Corollary 5. Let $R$ be an $A E$-ring such that $R^{2} \neq 0$. The following are equivalent:

(i) $R_{2}$ is a direct summand;

(ii) $R_{2}$ is an $A E$-ring and $R_{2}^{2} \neq 0$;

(iii) $R_{2}^{+}$is bounded;

(iv) every endomorphism on $R_{2}^{+}$extends to an endomorphism on $R^{+}$and $R_{2}^{2} \neq 0$.

Proof: (i) $\Rightarrow$ (ii) $\Rightarrow$ (iii) $\Rightarrow$ (i) with the last implication holding because a bounded, pure subgroup is a direct summand [6, Theorem 27.5]. Then (i) $\Rightarrow$ (iv) $\Rightarrow$ (ii) to complete the equivalences.

Corollary 6. Let $R$ be a ring with $R^{3} \neq 0$. Then $R$ is an $A E$-ring if and only if:

(i) $R=B \oplus N$, as a ring direct sum, with $B \cong F S(1)$,

(ii) $N^{2}=0=N_{2}$, and

(iii) $\operatorname{Hom}\left(N^{+}, C(2)\right)=0$. (Equivalently: $N^{+}$is 2-divisible.)

Proof: If $R$ is an $A E$-ring, then (i) and (ii) follow from [4, Theorem 5] and (iii) follows from Theorem 4 (iii). The converse is an immediate consequence of Theorem 4.

Feigelstock [4, Theorem 5] showed that an $A E$-ring $R$ with $R^{3} \neq 0$ satisfies (i) and (ii) of the above corollary. The authors have an alternate proof of this using a decomposition of self distributive rings $[2,9]$.

Example 7. Let $R=B \oplus N$ (ring direct sum).

(i) If $B$ is an $F S(n)$-ring and $N^{+}$is a divisible group such that $N_{2}=0$, then $R$ is an $A E$-ring.

(ii) If $B \cong Z /(2), N^{+} \cong C(4)$, and $N^{2}=0$, then $R$ is a self distributive, torsion, direct sum of $A E$-rings which is not an $A E$-ring.

(iii) If $B \cong Z /(2), N^{2}=0$, and $N^{+} \cong Z^{+}$, then $R$ is a self distributive, mixed, direct sum of $A E$-rings which is not an $A E$-ring.

This particular example shows that Feigelstock's characterisation of torsion $A E$-rings does not carry over to the nontorsion case, thus answering, in the negative, the "Question" posed in [4].

TheOREM 8. Let $R$ be an $A E$-ring such that $R^{3}=0$ and $R^{2} \neq 0$. Then either:

(i) $\quad R=R_{2} \oplus H$, where $H^{2}=0$ and $R_{2}=\Sigma \oplus A^{(i)}, i=1, \ldots, n$, where 
the additive group of each $A^{(i)}$ is a cyclic 2-group, $\left(A^{(i)}\right)^{2}=0$ and $\left|A^{(i)}\right|<\left|A^{(n)}\right|$ for each $i<n$, and $A^{(n)} \cong F S(m)$ for $m>1$; or

(ii) there is an ideal $X$ of $R$ which is the direct sum of a countably infinite collection of ideals $A^{(i)}$, where the additive group of each $A^{(i)}$ is a finite cyclic 2-group, $X R=0=R X$, and every finite sum of the $A^{(i)}$ is a direct summand of $R$.

Proof: Since $R^{2} \neq 0$, we have $R_{2}^{+}$is reduced [4, Theorem 4]. Using Corollary 27.3 of [6] repeatedly, we obtain $R=\left(\Sigma \oplus A^{(i)}\right) \oplus H^{(n)}, i=1, \ldots, n$, where the additive group of each $A^{(i)}$ is a finite cyclic 2-group. If for some $n, H_{2}^{(n)}=0$, the process terminates and $R_{2}=\Sigma \oplus A^{(i)}, i=1, \ldots, n$. In this case, using $R^{2} \neq 0$, $R^{3}=0$, and Theorem 6 in [4], yields that exactly one of the $A^{(i)}$ is a fundamental $A E$-ring, $F S(m)$, and all the other $A^{(i)}$ are square zero. Rearrange the terms in the direct sum so that the summand isomorphic to $F S(m)$ is the $n$th one. Lemma 2 then forces all the other $A^{(i)}$ to have order less than $2^{m}$.

If $H_{2}^{(n)} \neq 0$ for each $n$, then we obtain a countably infinite collection of $A^{(i)}$. If one of the $A^{(i)}$ is isomorphic to some $F S(m)$, remove it from the collection. (There is at most one $A^{(i)}$ isomorphic to a fundamental $A E$-ring by [4, Theorem 6].) The sum of the remaining collection is a direct sum of ideals; call it $X$. Observe that $X^{2}=0$. Any element in $X$ is in a finite sum of $A^{(i)}$ and hence is in a direct summand, which forces $X \subseteq \operatorname{Ann} R$.

Note that if $R$ is an $A E$-ring with $R^{3}=0$ and $R^{2} \neq 0$, if $R=A \oplus G \oplus M$, where $A \cong F S(n)$ and $G^{+} \cong C\left(2^{m}\right)$, then $n>m$ and $G^{2}=0$, by Lemma 2 .

Corollary 9. Let $R$ be an $A E$-ring satisfying

(i) $R^{3}=0$ and $R^{2} \neq 0$, and

(ii) either $R$ has a.c.c. on finite ideals contained in $R_{2}$, or $R$ has d.c.c. on ring direct summands.

Then $R$ is as in (i) of Theorem 8 .

Lemma 10. Let $R$ be an $A E$-ring with $R^{3}=0$.

(i) If $y \in R_{2}$ and $y$ has nonzero height in $R_{2}^{+}$, then $y \in \operatorname{Ann} R$.

(ii) If $y \in R_{2}$ and $y^{2} \neq 0$, then $y$ has height zero in $R_{2}^{+}$.

(iii) If $y \in R$ has order two, then $y \in \operatorname{Ann} R$.

Proof: (i) If $y=2 b$, then $y R=2 b R=0$; similarly $R y=0$.

(ii) Similar.

(iii) If $y \notin$ Ann $R$, then $y$ has height zero. By [6, Corollary 27.2], $R=C \oplus A$, where $C^{+}=(y)$. But this contradicts $R^{3}=0$. 
THEOREM 11. Let $R$ be an $A E$-ring satisfying:

(i) $R^{3}=0, R^{2} \neq 0, R^{+}$is reduced;

(ii) if $u \in R_{2}$ and $u \notin \operatorname{Ann} R$, then every element in (u) has finite height in $R^{+}$.

Then either $T\left(R^{+}\right) \subseteq$ Ann $R$; or $R=C \oplus A$, where $C \cong F S(n), n>1, C^{+}$is generated by an element of height zero in $R_{2}^{+}$, and $T\left(A^{+}\right) \subseteq$ Ann $R$. In the latter case, if $0 \neq x \in A_{2}$ and $(x)$ has no elements of infinite height in $A_{2}^{+}$, then $x$ has order less than $2^{n}$.

Proof: If $R_{2} \subseteq$ Ann $R$, then $T\left(R^{+}\right) \subseteq$ Ann $R$. Suppose $y \in R_{2}$ and $y \notin$ Ann $R$. Then $(y)$ is contained in a finite direct summand of $R^{+}$which is generated by an independent set of elements from $R_{2}$ [6, Corollary 27.9, Lemma 16.1]. At least one of the elements in this independent set is not in Ann $R$; so $R=C \oplus A$, where $C \cong F S(n),[4$, Theorem 6]. Lemma 10 (i) yields this generator of $C^{+}$has height zero. If $u \notin$ Ann $R$, $u \in A_{2}$, then in a similar manner to the above, we obtain $A=E \oplus A^{\prime}$, where $E^{+}$ is a cyclic 2-group generated by an element not in Ann $R$. But $R=C \oplus E \oplus A^{\prime}$; so by Theorem 6 of [4], $E^{2}=0$, a contradiction. Thus $A_{2} \subseteq$ Ann $R$ and consequently $T\left(A^{+}\right) \subseteq$ Ann $R$.

If $0 \neq x \in A_{2}$ and ( $x$ ) has no elements of infinite height in $A_{2}$, then $R=$ $C \oplus K \oplus A^{\prime \prime}$, where $K^{+}$is a finite direct sum of cyclic 2-groups [6, Corollary 27.9]. Each of these summands is a ring direct summand of $R$ and hence by Theorem 6 of [4] must have order less than $|C|=2^{n}$. Consequently $x$ has order less than $2^{n}$.

Corollary 12 . Let $R$ be an $A E$-ring satisfying:

(i) $R^{3}=0, R^{2} \neq 0$, and $R^{+}$is reduced;

(ii) $R_{2}^{+}$has no elements of infinite height;

(iii) $R_{2}$ is not contained in Ann $R$.

Then $R=C \oplus A$ as in Theorem 11 and $R_{2}^{+}=C^{+} \oplus F^{+}$, where $F^{+}$is the direct sum of cyclic groups each of order less than $2^{n}$.

Proof: To see that $F^{+}$has the desired properties, note that using Theorem 11 we have every element in $R_{2}$ has order less than or equal to $2^{n}$ and hence $R_{2}^{+}$is a direct sum of cyclic groups and each summand other than $C$ has order less than $2^{n}$. D

THEOREM 13. Let $R$ be an $A E$-ring.

(i) $R$ is an algebra over a field $K$ if and only if either $R^{2}=0$ or $R \cong Z /(2)$.

(ii) $R$ is indecomposable if and only if either

(a) $R^{2}=0$ and $R^{+}$is indecomposable, or

(b) $R \cong F S(n)$, for some $n \geqslant 1$.

(iii) $R$ is subdirectly irreducible if and only if either 
(a) $R^{2}=0$ and $R^{+} \cong C\left(p^{k}\right), 1 \leqslant p \leqslant \infty$, for some prime $p$, or

(b) $R \cong F S(n)$, for some $n \geqslant 1$.

Proof: Each part follows immediately from previous results and well-known properties of the additive groups involved.

THEOREM 14. Let $R$ be an $A E$-ring such that $R^{3} \neq 0$. If $S$ is a homomorphic image of $R$ such that $S_{2}$ is reduced, then $S$ is an $A E$-ring.

Proof: Let $f: R \rightarrow S$ be a surjective ring homomorphism. From Corollary 6 $S=f(B)+f(N)$. Either $f(B) \subseteq f(N)$, in which case $S^{2}=0$ and hence $S$ is an $A E$-ring, or $f(B) \cap f(N)=0$, in which case $S$ satisfies conditions (i) and (iii) of Corollary 6 and $(f(N))^{2}=0$. If $f(N)$ contains an element of order two, then its additive group has a cyclic 2-group as a direct summand [6, Corollary 27.3], and hence $N^{+}$maps homomorphically onto $C(2)$, a contradiction to (iii) of Corollary 6 . Thus $S=f(B) \oplus f(N)$ satisfies the hypotheses of Corollary 6 and $S$ is an $S E$-ring.

Observe that if $R$ is an $A E$-ring with $R^{3}=0$ and $S$ is a homomorphic image of $R$, then $S^{3}=0$ and $2 S^{2}=0$. A ring with the latter two properties need not be an $A E$-ring even if it is subdirectly irreducible, as the following example illustrates.

Example 15. Let $S$ be the vector space of 3 -tuples over the field $Z /(2)$ and define multiplication by $\left(a_{1}, b_{1}, c_{1}\right) \cdot\left(a_{2}, b_{2}, c_{2}\right)=\left(0,0, a_{1} b_{2}+b_{1} a_{2}\right)$. Then $S^{3}=2 S^{2}=0$, $x^{2}=0$ for each $x \in S$, and $S$ is a commutative, subdirectly irreducible ring (algebra over $Z /(2))$. But $S^{2} \neq 0$ and $S$ is not an $A E$-ring.

We close with some open questions and some observations.

QUeSTION I. Are all $A E$-rings commutative?

QUESTION II. Is every subdirectly irreducible homomorphic image of an $A E$-ring also an $A E$-ring?

QUeSTION III. Is every homomorphic image of an $A E$-ring an $A E$-ring?

Question IV. If $R$ is an $A E$-ring in which $x^{2}=0$ for each $x \in R$, is $R^{2}=0$ ?

Feigelstock's Theorem 6 [4] and several of our results herein show that for a wide class of $A E$-rings the answer to Question IV is "yes". An affirmative answer to any one of Questions I, II, III yields an affirmative answer to any prior question in the list.

\section{REFERENCES}

[1] G. Birkenmeier and H. Heatherly, 'Left self distributive near-rings', J. Austral. Math. Soc. (to appear).

[2] G. Birkenmeier, H. Heatherly and T. Kepka, 'Rings with left self distributive multiplication', (submitted). 
[3] S. Dhompongsa and J. Sanwong, 'Rings in which additive mappings are multiplicative', Studia Sci. Math. Hungar. 22 (1987), 357-359.

[4] S. Feigelstock, 'Rings whose additive endomorphisms are multiplicative', Period. Math. Hungar. 19 (1988), 257-260.

[5] S. Feigelstock, 'Rings whose additive endomorphisms are $n$-multiplicative', Bull. Austral. Math. Soc. 39 (1989), 11-14.

[6] L. Fuchs, Infinite Abelian Groups I (Academic Press, New York - London, 1970).

[T] I. Kaplansky, Infinite Abelian Groups (Univ. of Michigan Press, Ann Arbor, Michigan, 1954).

[8] K.H. Kim and F.W. Roush, 'Additive endomorphisms of rings', Period. Math. Hungar. 12 (1981).

[9] M. Petrich, 'Structure des demi-groupes et anneaux distributifs', C.R. Acad. Sci. Paris Sér. $A$ - B (1969), A849-A852.

[10] R.P. Sullivan, 'Research problems', Period. Math. Hungar. 8 (1977), 313-314.

\author{
Department of Mathematics \\ University of Southwestern Louisiana \\ Lafayette, Louisiana 70504 \\ United States of America
}

